\title{
Positional repeatability and variation in internal and external markers during volumetric-modulated arc therapy under end-exhalation breath-hold conditions for pancreatic cancer patients
}

\section{Makoto Sasaki}

Kyoto Daigaku Igakubu Fuzoku Byoin

Mitsuhiro Nakamura ( $\nabla$ m_nkmr@kuhp.kyoto-u.ac.jp )

Kyoto Daigaku Daigakuin Igaku Kenkyuka Igakubu https://orcid.org/0000-0001-5007-8808

Tomohiro Ono

Kyoto Daigaku Igakubu Fuzoku Byoin

Ryo Ashida

Kyoto Daigaku Igakubu Fuzoku Byoin

Michio Yoshimura

Kyoto Daigaku Igakubu Fuzoku Byoin

Manabu Nakata

Kyoto Daigaku Igakubu Fuzoku Byoin

Takashi Mizowaki

Kyoto Daigaku Daigakuin Igaku Kenkyuka Igakubu

Naozo Sugimoto

Kyoto Daigaku Daigakuin Igaku Kenkyuka Igakubu

\section{Research}

Keywords: Internal marker, external marker, breath-holding, kV X-ray monitoring, pancreatic cancer

Posted Date: March 25th, 2020

DOI: https://doi.org/10.21203/rs.3.rs-19263/v1

License: (c) (1) This work is licensed under a Creative Commons Attribution 4.0 International License.

Read Full License

Version of Record: A version of this preprint was published at Journal of Radiation Research on July 28th, 2020. See the published version at https://doi.org/10.1093/jrr/rraa054. 


\section{Abstract}

Background: To assess the positional repeatability of internal and external markers among multiple breath-hold $(\mathrm{BH})$ sessions and assess the positional variation of these markers within $\mathrm{BH}$ sessions for volumetric-modulated arc therapy (VMAT) for pancreatic cancer patients.

Methods: A total of 13 consecutive pancreatic cancer patients with an internal marker were enrolled. Single full-arc coplanar VMAT was delivered under end-exhalation (EE) BH conditions, while monitoring the internal marker with kilovoltage (kV) X-ray fluoroscopy. Positional repeatability of internal and external markers was indicated by the difference between the reference marker position and the marker position at the beginning of each $\mathrm{BH}$ session, and positional variation was indicated by the maximum displacement of the marker during each $\mathrm{BH}$ session.

Results: The overall positional repeatability was $0.6 \pm 1.5 \mathrm{~mm}$ in the X-axis for the centroid of the internal marker (ColM), $-0.1 \pm 2.2 \mathrm{~mm}$ in the $\mathrm{Y}$-axis for the ColM, and $0.8 \pm 2.2 \mathrm{~mm}$ for the external marker. An internal marker position $>2 \mathrm{~mm}$ from the reference position in the $\mathrm{Y}$-axis was observed in $19.1 \%$ of all $\mathrm{BH}$ sessions, despite the external marker position being $\leq 2 \mathrm{~mm}$ from the reference position. Meanwhile, the proportion of sessions with positional variation $\leq 2 \mathrm{~mm}$ was $93.2 \%$ and $98.7 \%$ for the ColM and external marker, respectively.

Conclusions: External marker motion can be used as a surrogate for pancreatic tumor motion during $\mathrm{BH}$ VMAT; however, an optimal internal margin is required to ensure positional repeatability.

\section{Background}

Radiotherapy is among the treatment options for patients with locally advanced unresectable pancreatic cancer (1). However, it is difficult to deliver a sufficient dose to the tumor because the pancreas is surrounded by radiosensitive organs at risk (OARs) of severe radiation-induced toxicity. Advanced techniques such as intensity-modulated radiation therapy (IMRT) and volumetric-modulated arc therapy (VMAT) are effective in reducing the dose to the OARs, while maintaining dose coverage to the tumor. Recently, VMAT has been increasingly used in clinical practice due to its ability to rapidly deliver beams (2).

Pancreatic tumors are known to move with respiration (3), which is an important consideration when treating pancreatic cancer with VMAT (4). Respiratory organ motion can result in underdosage of the target or overdosage of surrounding OARs (5). To address this problem, respiration motion management techniques, such as abdominal compression, breath-hold $(\mathrm{BH})$, respiratory gating, and real-time tumor tracking, have been used during the course of treatment (3). Of these techniques, $\mathrm{BH}$ is one of the most commonly used owing to its versatility (2). Some researchers reported that $\mathrm{BH}$ was clinically useful in cases with disease sites such as the lung, breast, liver, and pancreas (6-11). At our institution, VMAT has been used clinically under $\mathrm{BH}$ conditions at the end-exhalation $(\mathrm{EE})$ phase for pancreatic cancer $(9,12)$. 
When applying VMAT under EE-BH conditions for pancreatic cancer patients at our institution, an infrared (IR) reflective plastic box is placed on the abdominal surface as an external marker to monitor the patient's respiratory motion. Meanwhile, a gold coil (Visicoil; IBA, Louvain-la-Neuve, Belgium) is implanted around or inside the target as an internal surrogate. However, implantation is not always acceptable to the patient due to its invasiveness. Thus, it is preferable to estimate internal motion based on the external marker without implantation.

Several investigators have assessed positional uncertainties in pancreatic cancer under BH conditions (9-12). Nakamura et al. showed that the positional reproducibility of the pancreas under EE-BH conditions was $0.6 \pm 3.3 \mathrm{~mm}$ in the superior-inferior (SI) direction, using a visual feedback technique based on daily cone-beam computed tomography (CBCT) (9). Lens et al. concluded that the motion of pancreatic tumors under inhalation $\mathrm{BH}$ conditions was $4.2 \pm 2.3 \mathrm{~mm}$ in the SI direction, as shown by intratumoral fiducial markers and kilovoltage (kV) X-ray fluoroscopic images (10). Zeng et al. showed that the mean motion of an internal marker was $-0.6 \pm 2.3 \mathrm{~mm}$ in the SI direction, observed intermittently under inhalation $\mathrm{BH}$ conditions on $\mathrm{KV}$ X-ray images (11). However, there have been no reports assessing the positions of pancreatic tumors on a continuous basis, nor on the relationship between internal and external marker motion during VMAT under EE-BH conditions.

The aim of this study was to assess the positional repeatability of internal and external markers within multiple BH sessions, as well as the positional variation of the markers within BH sessions for VMAT under EE-BH conditions for pancreatic cancer patients. The results of this study could be used to determine the suitability of external marker motion as a surrogate for pancreatic tumor motion during BHs.

\section{Materials And Methods}

\section{Patients}

Among all pancreatic cancer patients who underwent VMAT under EE-BH conditions between July 2016 and July 2017, 13 consecutive patients with an internal marker were enrolled in this study (Table 1). As an internal marker, Visicoil (10-mm long and 0.5 or $0.75 \mathrm{~mm}$ in diameter) was endoscopically inserted into the tumor 1 to 2 weeks before treatment planning CT. More than $8 \mathrm{~mm}$ of three-dimensional (3D) internal marker motion was visually confirmed by a radiation oncologist using $\mathrm{kV} \mathrm{X}$-ray fluoroscopy under free breathing just before the treatment planning CT scan. All patients fasted for $>3$ hours and oral intake of water ceased 1 hour before the treatment planning CT scan and daily treatments. This study was performed in accordance with the Helsinki Declaration and was approved by our institutional review board (approval number: R1446). 
Table 1

Characteristics of patients treated for pancreatic cancer

\begin{tabular}{|c|c|c|c|c|c|}
\hline ID & Sex & Age $(y)$ & Tumor location & 3D tumor motion $(\mathrm{mm})$ & PTV-PRV volume $(\mathrm{mL})$ \\
\hline 1 & M & 77 & Body & 11.0 & 225.8 \\
\hline 2 & M & 85 & Head & 12.8 & 159.3 \\
\hline 3 & $\mathrm{~F}$ & 77 & Head & 13.0 & 164.8 \\
\hline 4 & $\mathrm{~F}$ & 48 & Head & 10.5 & 135.3 \\
\hline 5 & $M$ & 61 & Head & 20.8 & 220.8 \\
\hline 6 & $\mathrm{~F}$ & 86 & Head & 10.5 & 104.0 \\
\hline 7 & $\mathrm{~F}$ & 69 & Head & 12.0 & 366.9 \\
\hline 8 & $\mathrm{~F}$ & 79 & Head & 8.2 & 107.3 \\
\hline 9 & $\mathrm{~F}$ & 66 & Body & 11.0 & 119.7 \\
\hline 10 & $M$ & 64 & Head & 11.0 & 155.7 \\
\hline 11 & $\mathrm{~F}$ & 59 & Head & 10.9 & 176.2 \\
\hline 12 & $\mathrm{~F}$ & 55 & Head & 11.0 & 156.9 \\
\hline 13 & $\mathrm{~F}$ & 83 & Body & 10.4 & 145.8 \\
\hline
\end{tabular}

\section{CT scans and organ delineation}

All patients were immobilized by individualized vacuum pillows (Bofy Fix system; Elekta, Stockholm, Sweden) in the supine position with their arms raised. The breathing signal was acquired using an IR marker block attached to the abdominal surface of the patient and the Real-time Position Management System (RPM; Varian Medical Systems, Palo Alto, CA, USA) with a sample frequency of $30 \mathrm{~Hz}$. With respect to our institutional $\mathrm{BH}$ protocols, patients held their breath according to only the operator's coaching, without visual feedback; an operator instructed the patients to "breathe in and breathe out" while monitoring their breath signals using the RPM systems and to "hold their breath" at the timing of the end-exhalation. After several BH trainings, two planning CT scans with/without contrast-enhancement were performed under EE-BH conditions with a 16-slice CT scanner (LightSpeed RT16; GE Healthcare, Little Chalfont, UK). The CT scan range was from the superior border of the liver to the iliac crest, which took approximately 15 seconds, and then, the CT datasets were imported to the radiation treatment planning system (Eclipse version 13.7.14; Varian).

The gross tumor volume, clinical target volume (CTV), and OARs including the stomach, duodenum, intestine, liver, kidneys, and spinal cord were manually delineated on the CT images without contrast 
enhancement. The planning target volume (PTV) was determined by adding a 5-mm isotropic margin to the CTV. The planning organ at risk volume (PRV) was generated by adding around 3-5 $\mathrm{mm}$ of isotropic margin to the stomach, duodenum, and spinal cord. More detailed planning CT scan protocols and organ delineation were provided previously $(9,12,13)$.

\section{Treatment planning}

The single full-arc coplanar VMAT plan (gantry angle, $181-179^{\circ}$ in the clockwise direction) was created with the TrueBeam STx system (Varian) for all patients. The nominal energy and maximum dose rate were 10-megavolt (MV) flattening filter-free photon beams and 2,400 monitor units (MU)/min, respectively. A dose of 48 Gy in 15 fractions was prescribed in order to cover $95 \%$ of the PTV, subtracting the overlapping part of the PRVs. The plan isocenter was set to the center of the internal marker. The dose-volume constraints are shown in (14).

\section{Daily treatment procedure}

First, initial setup errors were corrected by reference to bony structures using an orthogonal kV X-ray imaging system (ExacTrac X-ray system, version 6.2.1; BRAINLAB, Feldkirchen, Germany). Subsequently, $\mathrm{CBCT}$ images were acquired under $\mathrm{EE}-\mathrm{BH}$ conditions while rotating the gantry angle by $200^{\circ}$ in two or three 20-s BHs, by interrupting the acquisition once or twice depending on the patient's BH ability. The $\mathrm{BH}$ protocols at the $\mathrm{CBCT}$ acquisition was performed by similar procedures as the planning $\mathrm{CT}$ scan. The final isocenter position was determined by correcting setup errors based on the internal maker positions in planning CT and CBCT images. Thereafter, the center position of the internal marker was monitored on kV X-ray fluoroscopic images (pixel size: $0.368 \mathrm{~mm}$ at isocenter, sample frequency: $25 \mathrm{~Hz}$ ), acquired with a gantry angle of approximately $90^{\circ}$ for a few seconds under EE-BH conditions using the $\mathrm{kV} \mathrm{X-ray} \mathrm{imager}$ of the TrueBeam STx system. Unless the internal marker position was within $\pm 2 \mathrm{~mm}$ from the plan isocenter, $\mathrm{BH}$ was repeated several times with monitoring of the $\mathrm{kV} \mathrm{X}$-ray fluoroscopic images to confirm the internal marker position. If the internal marker position was still more than $2 \mathrm{~mm}$ from the plan isocenter, CBCT images were reacquired under EE-BH conditions. This procedure was repeated until the internal marker position was within $\pm 2 \mathrm{~mm}$ from the plan isocenter on the $\mathrm{kV} \mathrm{X}$-ray fluoroscopic images under EE-BH conditions. This process was referred to as "positional verification".

After confirming that the internal marker position was within $\pm 2 \mathrm{~mm}$ from the plan isocenter, single fullarc coplanar VMAT was delivered under EE-BH conditions, while monitoring the internal marker using $\mathrm{kV}$ $\mathrm{X}$-ray fluoroscopy with a tube current of up to $7 \mathrm{~mA}$. The MV beam was delivered over two to five $\mathrm{BH}$ sessions (each approximately 15-30 seconds, depending on the patient's BH ability) for a single arc. When the center of the internal marker visually exceeded $2 \mathrm{~mm}$ from the plan isocenter on $\mathrm{kV} \mathrm{X}$-ray fluoroscopy for around 3 seconds, the MV beam was manually turned off. If the internal marker was within $\pm 2 \mathrm{~mm}$ of the plan isocenter again, the MV beam was manually turned on. This process was referred to as "MV beam delivery". Simultaneously, the IR marker block on the abdominal surface of the patient was monitored using the RPM system.

\section{Image processing}


The centroid of the internal marker (ColM) was detected on the kV X-ray fluoroscopic images, acquired during $\mathrm{BH}$ using in-house software programmed in the MATLAB environment (version 2017b; MathWorks Inc., Natick, MA, USA).

\section{Positional uncertainties}

Figure 2 shows the definitions of positional uncertainties of the ColM and external markers. Positional repeatability was given by the difference between the reference and the following $\mathrm{BH}$ session internal and external marker positions, for both positional verification and MV beam delivery. The reference position of the ColM was represented as a distance from the plan isocenter, which was set to zero position. The reference position of the external marker was represented as a distance from the zero position, which was defined by the lowest position in the first respiratory cycle of the RPM signals under free breathing reacquired after CBCT scan. Positional variation was defined as the absolute displacement from the reference marker position during each $\mathrm{BH}$ session under $\mathrm{MV}$ beam delivery.

\section{Data analysis}

The means and standard deviations (SDs) of the CoIM for positional repeatability were calculated for each patient in the $\mathrm{X}$ - and $\mathrm{Y}$-axis of the fluoroscopic images, and those of external marker motion were calculated in the AP direction. Similarly, the means and SDs of the CoIM for positional variation were calculated for each patient in the $\mathrm{Y}$-axis of fluoroscopic images, and those of the external marker motion were calculated in the AP direction.

In this study, intra- and inter-fractional subanalyses were also conducted. Three or more BH sessions were commonly required on a treatment day. As intra-fractional subanalyses, each treatment day was divided into four sessions: 1st $\mathrm{BH}$ session, 2nd BH session, 3rd BH session, and other $\mathrm{BH}$ sessions. Positional repeatability and variation were then assessed for the individual $\mathrm{BH}$ sessions. In addition, the treatment course was divided into three 5-day treatment sections to see how many differences occurred between three sections. Positional repeatability and variation were then assessed by section; details are shown in Fig. 2. One-way analysis of variance (ANOVA) was performed to evaluate differences in positional repeatability and positional variation, by $\mathrm{BH}$ session and by 5 -day treatment section. Tukey's multiple comparison test was performed when ANOVA showed significant differences. P-values $<0.05$ indicated a significant difference in both tests.

Pearson's correlation coefficients ( $r$ ) between the ColM motions in the Y-axis and the external marker motions in the AP direction during each $\mathrm{BH}$ session were calculated. These relationships were evaluated based on the following categorization: Weak correlation, absolute correlation coefficient, $|r|<0.4$; moderate correlation, $0.4 \leq|r|<0.7$; and strong correlation, $|r| \geq 0.7$. A regression coefficient (a) was also calculated. When the regression coefficient was larger than 1, the internal marker displacement was larger than the external marker displacement. Based on these datasets, the relationship between the CoIM and the external marker positions was assessed to determine whether external marker motion could be used 
as a surrogate for internal marker motion during $\mathrm{BHs}$. In this study, a threshold of external marker motion was set to $\pm 2 \mathrm{~mm}$ from the reference position as well as that of internal marker motion.

\section{Results}

\section{Marker detection}

Data on a total of $1,235 \mathrm{BH}$ sessions from 13 patients were obtained, including both positional verification and MV beam delivery, during the overall treatment course. In addition, the first kV X-ray fluoroscopic image of each $\mathrm{BH}$ session was used in the analysis of positional repeatability.

Based on the analysis of positional variation, three patients (patient 5,10 , and 11) were excluded from the analysis due to the extreme difficulty in detecting the ColMs. Consequently, $697 \mathrm{BH}$ sessions (10 patients) under MV beam delivery were used to analyze positional variation, encompassing a total of 260,015 datasets, all consisting of $\mathrm{kV} X$-ray fluoroscopic images and the corresponding external marker positions. Ultimately, the ColM was detected on $157,754 \mathrm{kV}$ X-ray fluoroscopic images (60.7\%), with some of the data being excluded due to low image contrast according to the gantry angle.

\section{Positional repeatability and variation throughout the treatment course}

Table 2 shows the positional repeatability and variation for the CoIM and the external marker. The overall positional repeatability was $0.6 \pm 1.5,-0.1 \pm 2.2 \mathrm{~mm}$, and $0.8 \pm 2.2 \mathrm{~mm}$, in the X-axis for the ColM, in the Yaxis for the ColM, and in the AP direction for the external marker, respectively. In total, $19.1 \%$ of the 1,235 $\mathrm{BH}$ sessions showed positional repeatability $>2 \mathrm{~mm}$ in the $\mathrm{Y}$-axis for the ColM, and $\leq 2 \mathrm{~mm}$ for the external marker. 
Table 2

Positional repeatability among $\mathrm{BH}$ sessions and positional variation within $\mathrm{BH}$ sessions for all patients.

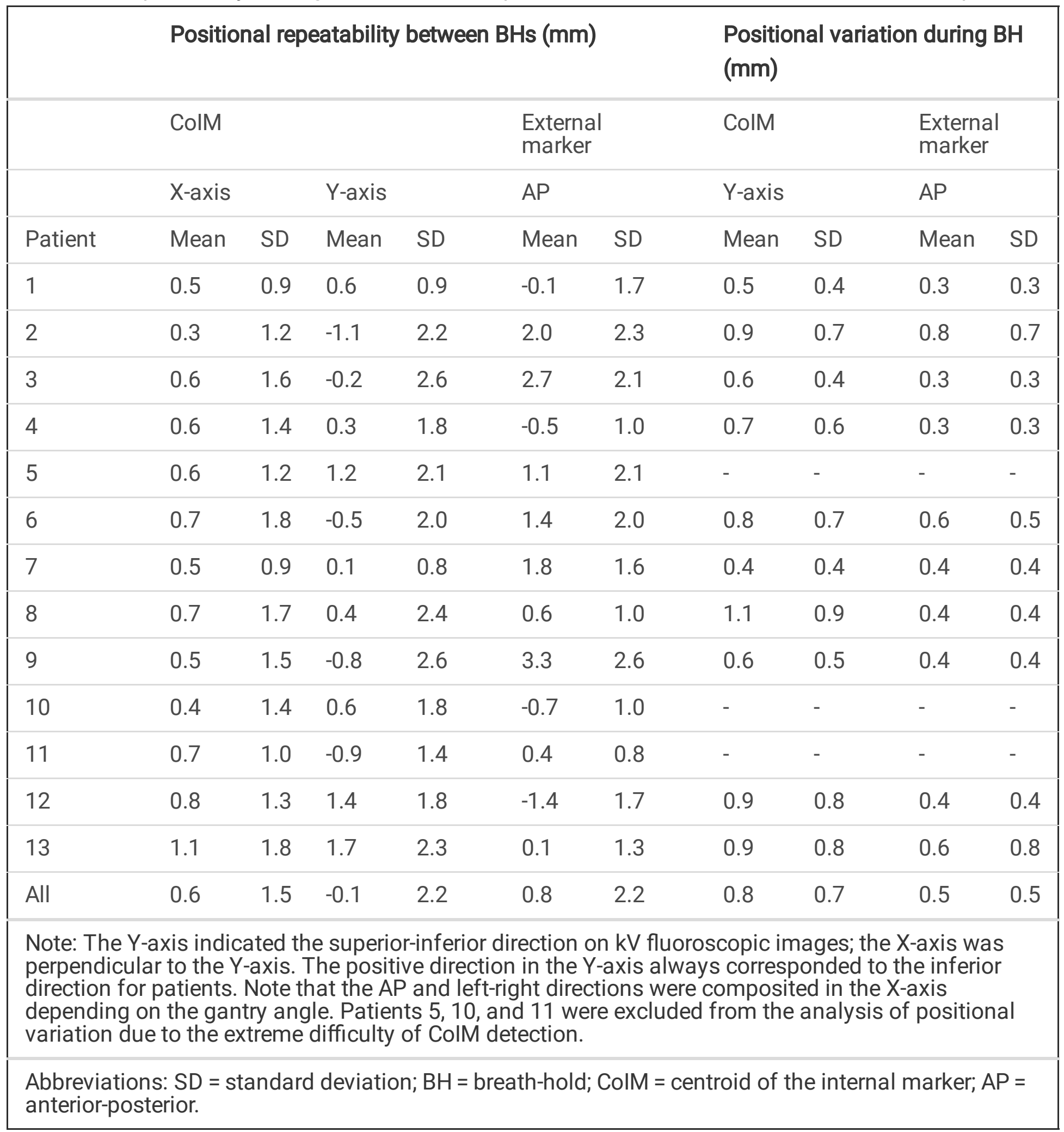

The overall positional variation was $0.8 \pm 0.7 \mathrm{~mm}$ in the $\mathrm{Y}$-axis for the ColM, and $0.5 \pm 0.5 \mathrm{~mm}$ in the AP direction for the external marker. The proportions of absolute positional variation $\leq 2 \mathrm{~mm}$ was $93.2 \%$ and $98.7 \%$ for the ColM and external marker, respectively. Moreover, $93.8 \%$ of the internal marker 
displacement was within $\pm 2 \mathrm{~mm}$ from the reference position when the external marker displacement was $\leq 2 \mathrm{~mm}$ (from the reference position).

\section{Intra- and inter-fractional subanalyses of positional repeatability and positional variation}

In the intra-fractional subanalyses, there was no significant difference in positional repeatability between the 1 st $\mathrm{BH}$ session group and the other groups, for both ColM and external markers, during the course of treatment [Fig. 3(a, b, c)]. Meanwhile, the ANOVA showed significant differences in positional variation within BH sessions ( $\mathrm{p}$ 0.05), and Tukey's multiple comparison test showed significant group differences for the ColM in the $\mathrm{Y}$-axis and the external marker, with the exception of the comparison between the 2nd and 3rd BH sessions for the external marker [Fig. 3(d, e)]. The mean positional variation for the ColM was $0.81 \mathrm{~mm}$ (95\% confidence interval [Cl], 0.80 to 0.82) in the 1 st $\mathrm{BH}$ session, $0.74 \mathrm{~mm}(95 \% \mathrm{Cl}, 0.73$ to 0.75$)$ in the 2nd $\mathrm{BH}$ session, $0.69 \mathrm{~mm}(95 \% \mathrm{Cl}, 0.68$ to 0.70$)$ in the $3 \mathrm{rd} \mathrm{BH}$ session, and $0.88 \mathrm{~mm}(95 \% \mathrm{Cl}, 0.87$ to 0.89 ) for the other $\mathrm{BH}$ sessions. Positional variation decreased gradually from the $1 \mathrm{st}$ to the $3 \mathrm{rd} \mathrm{BH}$ session; however, variation increased again in the group with more than $3 \mathrm{BH}$ sessions [Fig. 3(d)].

In the inter-fractional subanalyses, ANOVA showed significant differences in positional repeatability $(p<$ $0.05)$ in the $\mathrm{Y}$-axis for the CoIM, and in the positional variation $(\mathrm{p}<0.05)$ of both the internal and external markers. The ANOVA showed no significant differences in positional repeatability in the X-axis for the ColM or the external marker [Fig. 4(a, c)]. Meanwhile, Tukey's multiple comparison test showed significant differences in positional repeatability in the $\mathrm{Y}$-axis for the ColM between the 1st 5-day treatment section (mean, $0.48 \mathrm{~mm}[95 \% \mathrm{Cl}, 0.30$ to 0.67$]$ ) and the $3 \mathrm{rd}$ 5-day treatment section (mean, $0.05 \mathrm{~mm}[95 \% \mathrm{Cl},-0.12$ to 0.22 ]) $(p<0.05)$ [Fig. 4(b)]. Additionally, there was a significant difference in positional variation between the 1st 5-day treatment section and the other sections $(p<0.05)$; however, no significant difference between the 2nd 5-day treatment section and the 3rd 5-day treatment section was observed in the $\mathrm{Y}$-axis for the CoIM [Fig. 4(d)]. The mean positional variation was $0.81 \mathrm{~mm}(95 \% \mathrm{Cl}, 0.80$ to 0.82$)$ in the 1 st 5 -day treatment section, $0.80 \mathrm{~mm}(95 \% \mathrm{Cl}, 0.79$ to 0.81$)$ in the 2 nd 5 -day treatment section, and $0.71 \mathrm{~mm}(95 \% \mathrm{Cl}, 0.70$ to 0.72$)$ in the $3 \mathrm{rd}$ 5-day treatment section.

\section{Correlation between the internal and external markers}

In all the $\mathrm{BH}$ sessions, the correlation between the ColM and external marker positions showed a weak relationship in 54\%, a moderate relationship in 30\%, and a strong relationship in $16 \%$ of the sessions [Fig. 5(a)]. For BH sessions with the weak correlation, the percentage of positional variations where both markers were within $2 \mathrm{~mm}$ was $96.1 \%$. Meanwhile, $60 \%$ of the $\mathrm{BH}$ sessions where positional variation of the external marker was greater than $\pm 2 \mathrm{~mm}$ (from the reference position) showed a strong correlation between the ColM and external marker positions [Fig. 5(b)]. Moreover, 70\% of the BH sessions in which external marker motion was greater than $\pm 2 \mathrm{~mm}$, and there was a strong correlation between the ColM and external marker positions, had a regression coefficient greater than 1 [Fig. 5(c)]. Figure 6(a, b) shows an example case (patient 8) with a weak correlation between the internal and external marker motions within $\mathrm{BH}$ sessions, due to a slow drift in the external marker position. Figure $6(\mathrm{c}, \mathrm{d})$ shows another 
example case (patient 4), in which there was a strong correlation between the internal and external marker motions within $\mathrm{BH}$ sessions, which was due to sudden motion of both markers in the positive direction 6 seconds after the $\mathrm{BH}$ phase.

\section{Discussion}

We assessed internal and external marker motions during VMAT under EE-BH conditions for pancreatic cancer patients. Lens et al. reported a mean positional repeatability between consecutive BHs during the inhalation phase of $-0.5 \pm 0.8$ and $-0.2 \pm 1.7 \mathrm{~mm}$ in the AP and SI direction, respectively, for pancreatic cancer patients (as indicated by the Visicoil marker) (10). In the present study, the overall positional repeatability for the CoIM was $0.6 \pm 1.5$ and $-0.1 \pm 2.2 \mathrm{~mm}$ in the $\mathrm{X}$ - and $\mathrm{Y}$-axis, respectively. Regarding other disease sites, Lu et al. investigated the positional repeatability between BHs for eight liver and seven lung cancer patients under active breathing conditions, by comparing three consecutive CT scans (6). For liver cancer patients, the repeatability of systematic and random errors was 1.4 and $1.0 \mathrm{~mm}$ in AP direction and 1.6 and $1.4 \mathrm{~mm}$ in SI direction, respectively; for lung cancer patients, the respective values were 1.5 and $1.1 \mathrm{~mm}$ in the AP direction and 4.0 and $1.9 \mathrm{~mm}$ in the SI direction. Although the disease sites were different from ours, our $\mathrm{BH}$ approach showed comparable positional repeatability with active breathing control.

Regarding positional repeatability, the ColM in the Y-axis $>2 \mathrm{~mm}$ was observed in $19.1 \%$ of all $\mathrm{BH}$ sessions, despite the external marker position being $\leq 2 \mathrm{~mm}$. Based on these results, it is difficult to estimate the positional repeatability of the tumor only by referencing the position of the external marker during each $\mathrm{BH}$ session; an optimal internal margin is thus required to ensure positional repeatability of a pancreatic tumor without an internal marker.

Regarding positional variation, we found that both the internal and external markers drifted, even during BH [Fig. 6(a, c)]. According to Takao et al., this could be due mainly to physiological movements such as pulsations and muscle relaxation, or an incomplete $\mathrm{BH}(15)$. Meanwhile, the correlation between internal and external marker motions during BH was weak in a majority (51\%) [Fig. 5(a)], which may be represented the physiological movements. Of these $\mathrm{BH}$ sessions, most of the positional variations of both markers were within $2 \mathrm{~mm}$. From these results, we confirmed that most of the internal marker displacement can be ensured to be within $2 \mathrm{~mm}$ as long as one monitors that the external marker displacement is $<2 \mathrm{~mm}$, even in a $\mathrm{BH}$ session with a weak correlation. In contrast, $60 \%$ of $\mathrm{BH}$ sessions where external marker displacement exceeded $2 \mathrm{~mm}$ (from the reference position) showed a strong correlation between the internal and external marker motions [Fig. 5(b)]. This might represent the incomplete $\mathrm{BH}$, as a transition to inhalation phase due to limits of the $\mathrm{BH}$. In the $\mathrm{BH}$ sessions with a strong correlation between the internal and external markers, monitoring the external marker motions during the $\mathrm{BH}$ could predict motion of the pancreatic tumor, as reported by Shen et al. (16); therefore, we are convinced that external marker motion can generally be used as a surrogate for internal marker motion during $\mathrm{BH}$, despite a correlation coefficient. 
In the intra-fractional subanalyses, the positional variation gradually decreased from the 1 st to $3 \mathrm{rd} \mathrm{BH}$ session, while the variation increased in the group with more than $3 \mathrm{BH}$ sessions [Fig. 3 (d, e)]. In the interfraction subanalyses, positional variation improved during the 3rd 5-day treatment section (day 11-15), compared to that during the 1st 5-day treatment section (day 1-5). According to Lee et al., BH training before treatment improved the positional consistency of lung tumors (17). To further improve the positional consistency of pancreatic cancer motion during the 1st $\mathrm{BH}$ session within the first few days after beginning treatment, sufficient training of the patient in $\mathrm{BH}$ would be useful.

One limitation of this study was that positional variation was analyzed only in the SI direction because the motion magnitudes in the AP and LR directions were unclear due to the dependence on the gantry angle. Whitfield et al. reported that the inter- and intra-fractional pancreatic cancer motion was larger in the SI direction than in other directions under free breathing conditions (18), and Lens et al. reported that pancreatic cancer motion under inhalation-BH conditions was greater in the SI direction than in the AP direction (10); therefore, we assume that positional repeatability and variation would be smaller in the AP and LR directions than in the SI direction.

According to Nakamura et al., positional repeatability of $>5 \mathrm{~mm}$ for the pancreas was occasionally observed in daily CBCT, even when using visual feedback (9). In addition, the usefulness of visual feedback for positional variation during $\mathrm{BH}$ has not been reported. Considering these aspects, our institutional BH protocols are implemented without visual feedback, which may be another limitation. In contrast, several studies have reported that visual feedback provided high repeatability of $\mathrm{BH}(12,17,19)$. Given the visual feedback, it may have improved the positional repeatability and variation.

\section{Conclusions}

We assessed the positional repeatability among multiple $\mathrm{BH}$ sessions, and the positional variation within $\mathrm{BH}$ sessions, of internal and external marker motions during the course of treatment for pancreatic cancer patients. We found that external marker motion can generally be used as a surrogate for pancreatic tumor motion during $\mathrm{BH}$; however, an internal marker position $>2 \mathrm{~mm}$ from the reference position in the $\mathrm{Y}$-axis was observed in $19.1 \%$ of all BH sessions, despite the external marker position being $\leq 2 \mathrm{~mm}$ from the reference position. In addition, we found that positional variation of the internal marker during $\mathrm{BH}$ was larger for BHs within the first few days of treatment.

\section{Abbreviations}

3D: three-dimensional; ANOVA: analysis of variance; AP: anterior-posterior; BH: breath-hold; CBCT: conebeam computed tomography; CoIM: centroid of the internal marker; CTV: clinical target volume; EE: endexhalation; IMRT: intensity-modulated radiation therapy; IR: infrared; kV: kilovoltage; LR: left-right; MU: monitor unit; MV: megavolt; OAR: organ at risk; RPM: Real-time Position Management; PRV: planning organ at risk volume; PTV: planning target volume; SD: standard deviation; VMAT: volumetric-modulated arc therapy. 


\section{Declarations}

\section{Ethical Considerations}

This study was performed in accordance with the Helsinki Declaration and approved by our institution review board (approval number: R1446).

\section{Consent for publication}

We got consent using our institution form.

\section{Availability of data and materials}

The datasets supporting the conclusions of this article are available from the corresponding author on reasonable request.

\section{Competing interest}

The authors declare that they have no competing interests.

\section{Fundings}

This research was supported in part by JSPS KAKENHI (Grant Nos. 18H02766 and 18K15545).

\section{Author contributions}

MS and MN designed the study. MS collected data and wrote the initial draft of the manuscript. MN contributed to analysis and interpretation of data and assisted in the preparation of the manuscript. All other authors contributed to analysis and interpretation of data and critically reviewed the manuscript. All authors read and approved the final manuscript.

\section{References}

1. Tempero MA, Malafa MP, Al-Hawary M, Asbun H, Bain A, Behrman SW, et al. Pancreatic adenocarcinoma, version 2.2017, NCCN clinical practice guidelines in oncology. J Natl Compr Cancer Netw. 2017;15:1028-61.

2. Akino $Y$, Tohyama N, Akita $K$, Ishikawa $M$, Kawamorita $R$, Kurooka $M$, et al. Modalities and techniques used for stereotactic radiotherapy, intensity-modulated radiotherapy, and image-guided radiotherapy: A 2018 survey by the Japan Society of Medical Physics. Phys Med. 2019;64:182-7.

3. Keall P, Mageras G, Balter J, Emery R, Forster K, Jiang S, et al. The management of respiratory motion in radiation oncology report of AAPM Task Group 76. Med Phys. 2006;33:3874-900.

4. Akimoto M, Nakamura M, Nakamura A, Mukumoto N, Kishi T, Goto Y, et al. Inter- and intrafractional variation in the 3-dimensional positions of pancreatic tumor due to respiration under real-time monitoring. Int J Radiat Oncol Biol Phys. 2017;98:1204-11. 
5. Sasaki M, Nakamura M, Mukumoto N, Goto Y, Ishihara Y, Nakata M, et al. Variation in accumulated dose of volumetric-modulated arc therapy for pancreatic cancer due to different beam starting phase. J Appl Clin Med Phys. In press.

6. Lu L, Diaconu C, Djemil T, Videtic GMM, Abdel-Wahab M, Yu N, et al. Intra- and inter-fractional liver and lung tumor motions treated with SBRT under active breathing control. J Appl Clin Med Phys. 2015;19:39-45.

7. Osman S, Hol S, Poortmans P, Essers M. Volumetric modulated arc therapy and breath-hold in imageguided locoregional left-sided breast irradiation. Radiother Oncol. 2014;112:17-22.

8. Lin Y, Ozawa S, Miura H, Yogo K, Nakashima T, Miki K, et al. Split-VMAT technique to control the expiratory breath-hold time in liver stereotactic body radiation therapy. Phys Med. 2017;40:17-23.

9. Nakamura M, Akimoto M, Ono T, Nakamura A, Kishi T, Yano S, et al. Interfraction positional variation in pancreatic tumor using daily breath-hold cone-beam computed tomography with visual feedback. J Appl Clin Med Phys. 2015;16:108-16.

10. Lens $E$, van der Horst $A$, Versteijne $E$, Bel $A$, van Tienhoven G. Considerable pancreatic tumor motion during breath-holding. Acta Oncol. 2016;55:1360-8.

11. Zeng C, Xiong W, Li X, Reyngold M, Gewanter R, Cuaron J, et al. Intrafraction tumor motion during deep inspiration breath hold pancreatic cancer treatment. J Appl Clin Med Phys. 2019;20:37-43.

12. Nakamura M, Shibuya K, Shiinoki T, Matsuo Y, Nakamura A, Nakata M, et al. Positional reproducibility of pancreatic tumors under end-exhalation breath-hold conditions using a visual feedback technique. Int J Radiat Oncol Biol Phys. 2011;79:1565-71.

13. Goto Y, Nakamura A, Ashida R, Sakanaka K, Itasaka S, Shibuya K, et al. Clinical evaluation of intensity-modulated radiotherapy for locally advanced pancreatic cancer. Radiat Oncol. 2018;13:1-9.

14. Ziegler M, Nakamura M, Hirashima H, Ashida R, Yoshimura M, Bert C, et al. Accumulation of the delivered treatment dose in volumetric modulated arc therapy with breath-hold for pancreatic cancer patients based on daily cone beam computed tomography images with limited field-of-view. Med Phys. 2019;46:2969-77.

15. Takao S, Miyamoto N, Matsuura T, Onimaru R, Katoh N, Inoue T, et al. Intrafractional baseline shift or drift of lung tumor motion during gated radiation therapy with a real-time tumor-tracking system. Int J Radiat Oncol Biol Phys. 2016;94:172-80.

16. Shen Z, Andrews M, Balik S, Magnelli A, Setphans K, Videtic G, et al. Real-time position management (RPM) system as a valuable tool to predict tumor position deviation in SBRT lung and liver patients with breath hold using active breathing coordinator (ABC). Med Phys. 2016;43:3440.

17. Lee D, Greer P, Lapuz C, Ludbrook J, Hunter P, Arm J, et al. Audiovisual biofeedback guided breathhold improves lung tumor position reproducibility and volume consistency. Adv Radiat Oncol. 2017;2:354-62.

18. Whitfield G, Jain P, Green M, Watkins G, Henry A, Stratford J, et al. Quantifying motion for pancreatic radiotherapy margin calculation. Radiother Oncol. 2012;103:360-6. 
19. Goossens S, Senny F, Lee J, Janssens G, Geets X. Assessment of tumor motion reproducibility with audio-visual coaching through successive 4D CT sessions. J Appl Clin Med Phys. 2014;15:47-56.

\section{Figures}

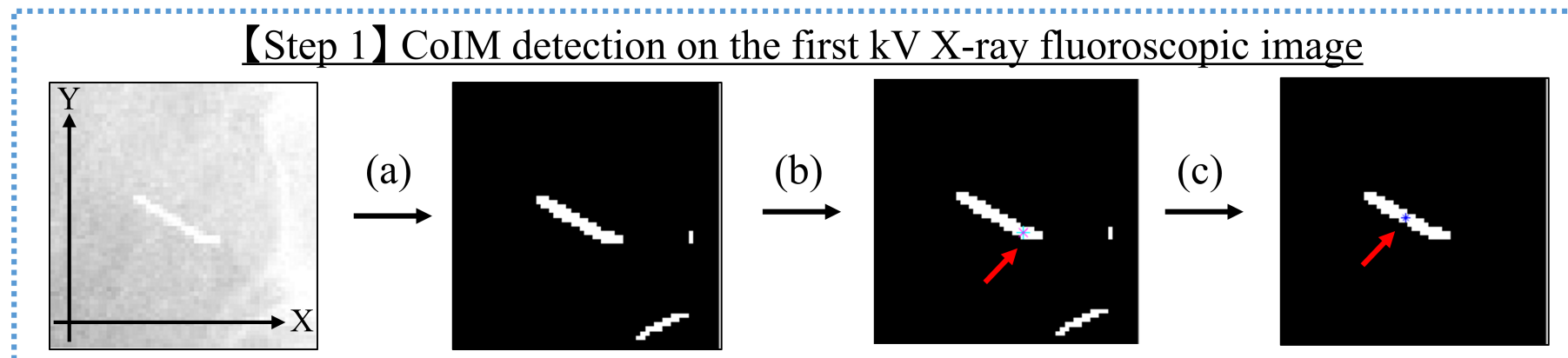

(a) Binarization and filterization, (b) Select marker (red arrow), (c) Automatic detection of the CoIM (red arrow)

\section{【Step 2】 CoIM detection on all kV X-ray fluoroscopic images}

Exclusion of incorrect CoIMs (red arrow)

(d) Automatic exclusion

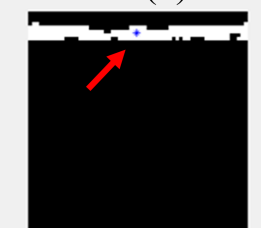

(e) Manual exclusion

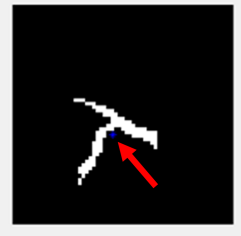

$\sqrt{+}, \sqrt{n}$
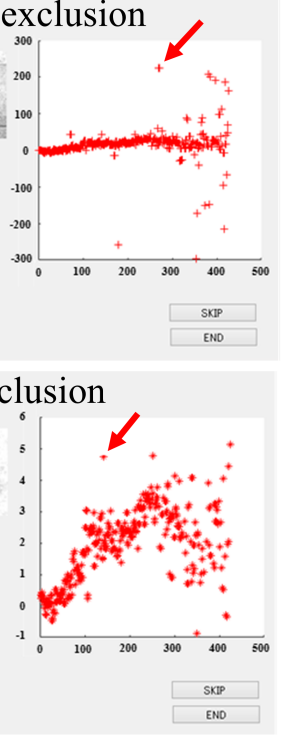

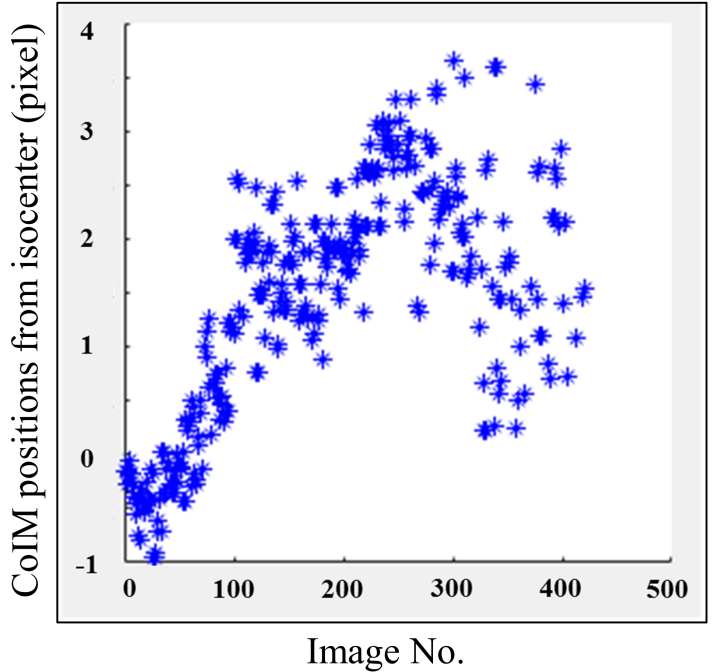

Image No.

\section{Figure 1}

Procedure for detecting the CoIM. In Step 1, the ColM is detected on the first kV X-ray fluoroscopic image. The fluoroscopic image was binarized and had a Hessian-based multiscale filter applied (a). Manual selection of the internal marker (b) and automatic detection of the CoIM (c). In Step 2, the algorithm automatically detected the CoIM on all kV X-ray fluoroscopic images. Thereafter, images with incorrect ColMs were excluded automatically (d) and manually (e). Abbreviations: ColM, centroid of the internal marker; kV, kilovoltage; AP, anterior-posterior; SI, superior-inferior; LR, left-right. 
Inter-fractional subanalysis

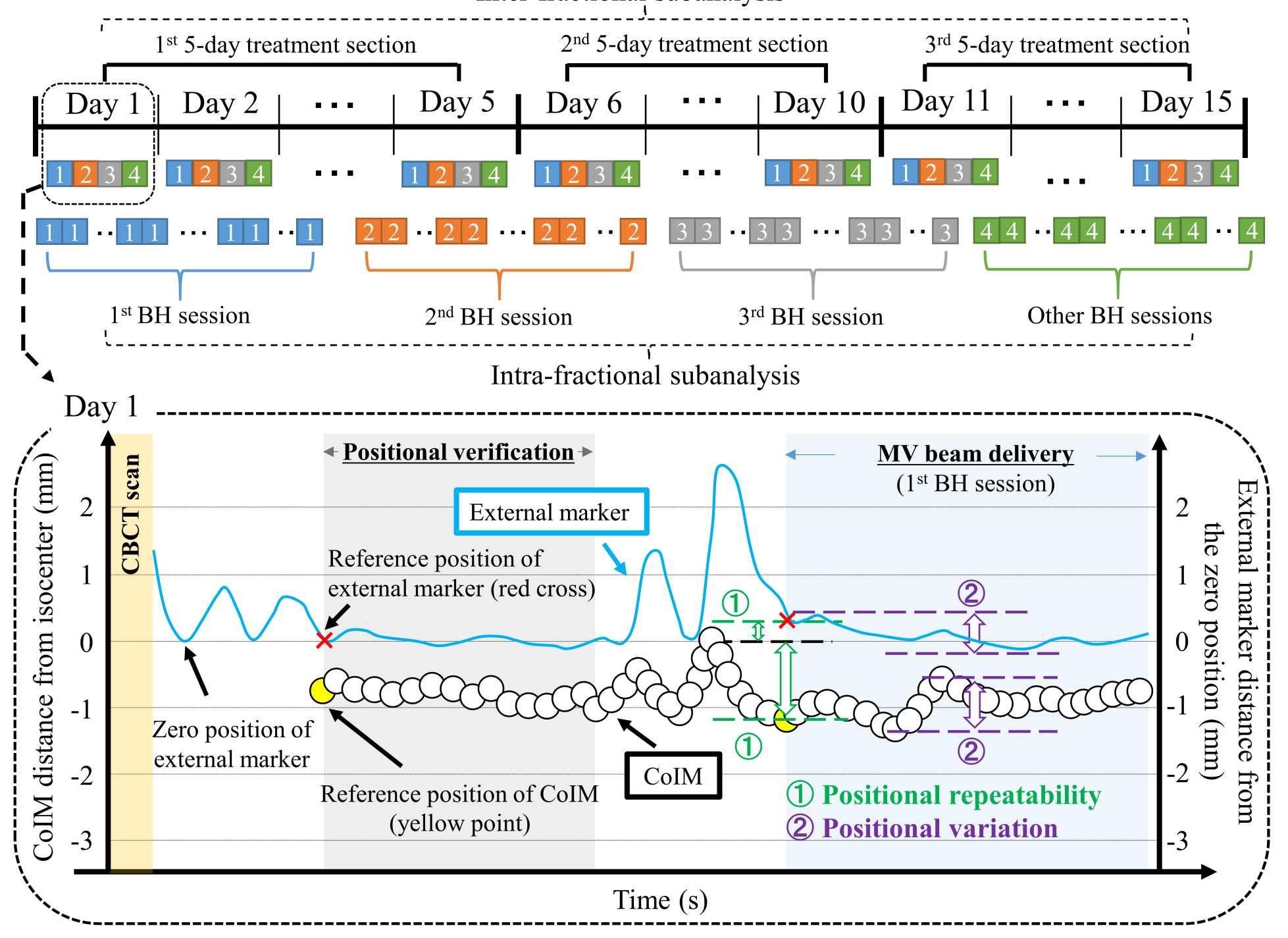

Figure 2

Positional uncertainties of the ColM and external marker. Intra-fractional subanalyses were performed, and each treatment day was divided into four sessions. Inter-fractional subanalyses were performed where the treatment course was divided into three 5-day treatment sections. Zero position of the external marker was defined by the lowest position in the first respiratory cycle in the RPM signals under free breathing, reacquired after $\mathrm{CBCT}$ scan. Abbreviations: $\mathrm{BH}$, breath-hold; $\mathrm{EE}$, end-exhalation. 


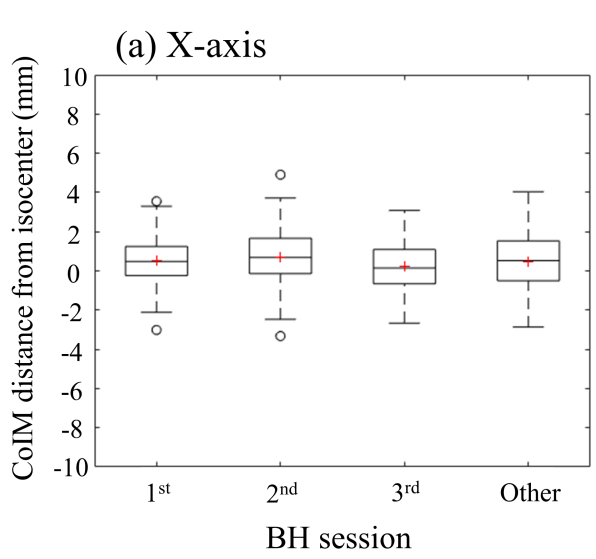

(d) Y-axis for the CoIM

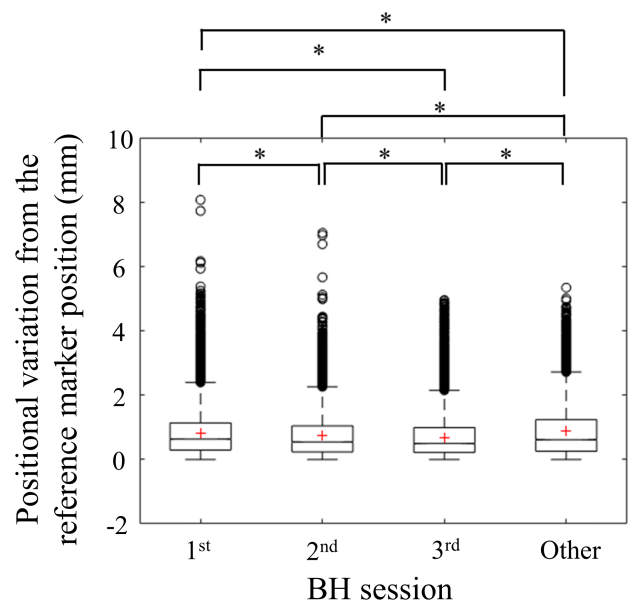

(b) Y-axis

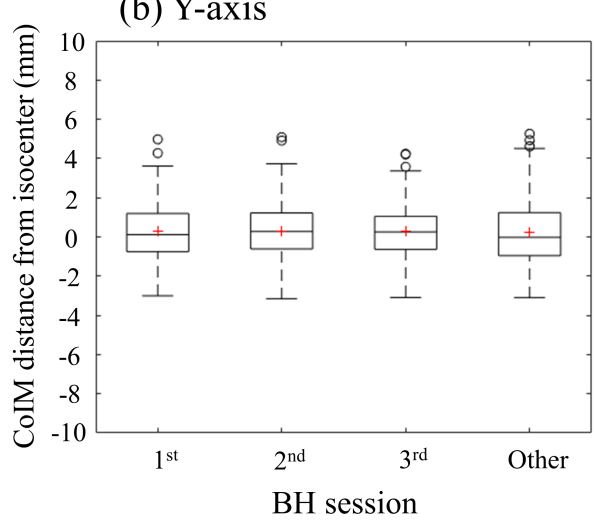

(e) External marker
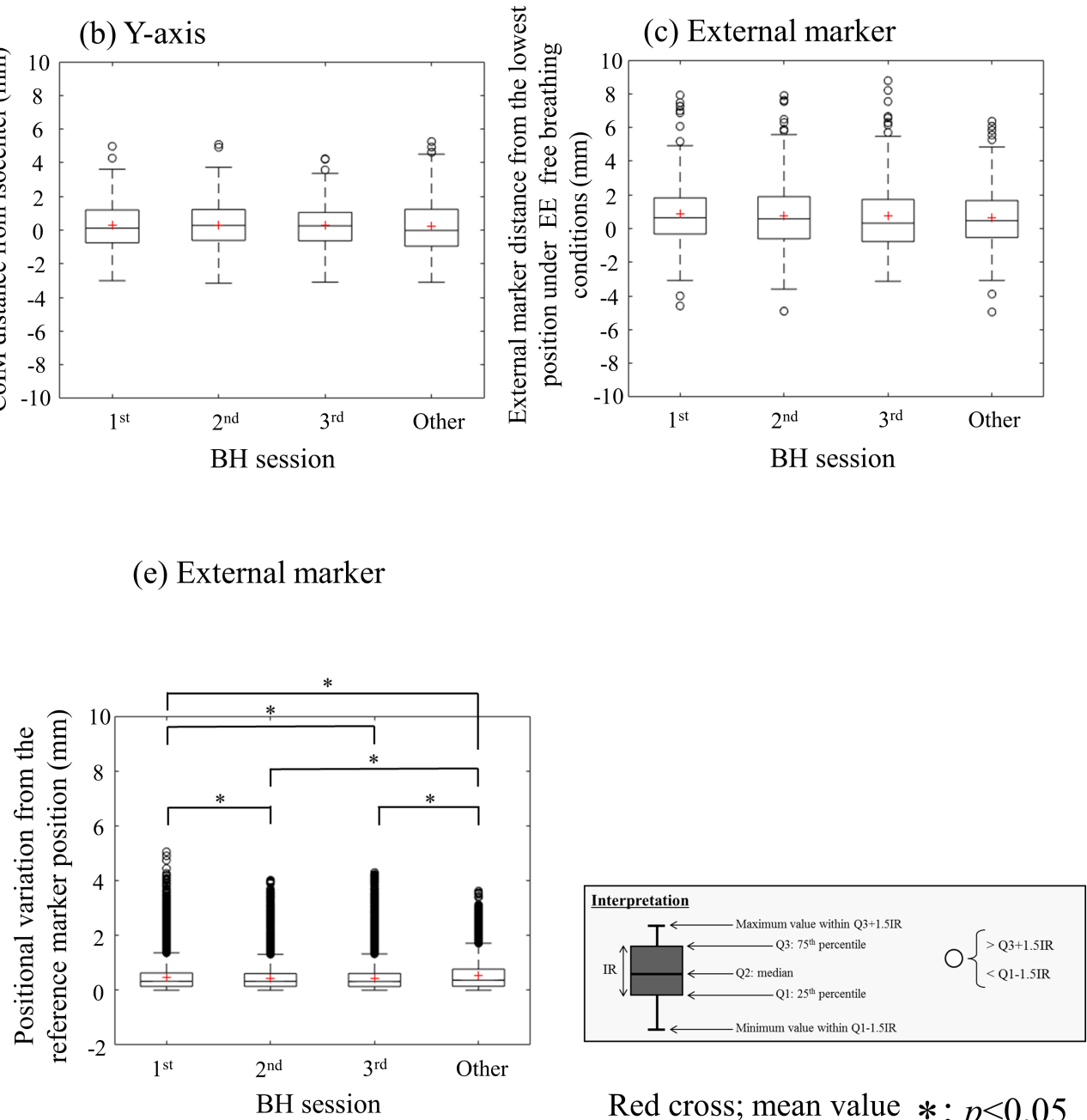

Red cross; mean value $* ; p<0.05$

\section{Figure 3}

Box-and-whisker plots of the intra-fractional subanalyses of positional repeatability among multiple $\mathrm{BH}$ sessions and positional variation within $\mathrm{BH}$ sessions, for all patients. The positional repeatability among $\mathrm{BH}$ sessions for the ColM on the $\mathrm{X}$-axis (a) and on the $\mathrm{Y}$-axis (b), and for the external marker (c). The positional variation within $\mathrm{BH}$ sessions for the ColM on the $\mathrm{Y}$-axis (d), and for the external marker (e). The SI direction for patients on fluoroscopic images corresponded to the $\mathrm{Y}$-axis; the $\mathrm{X}$-axis was perpendicular to the $Y$-axis. 


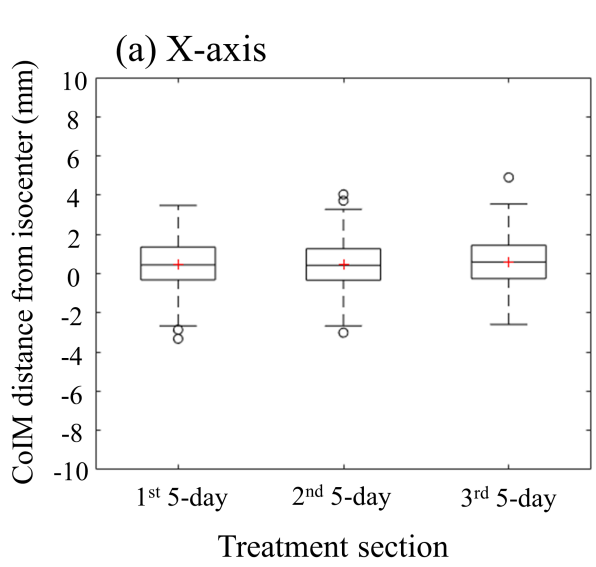

(d) Y-axis for the CoIM

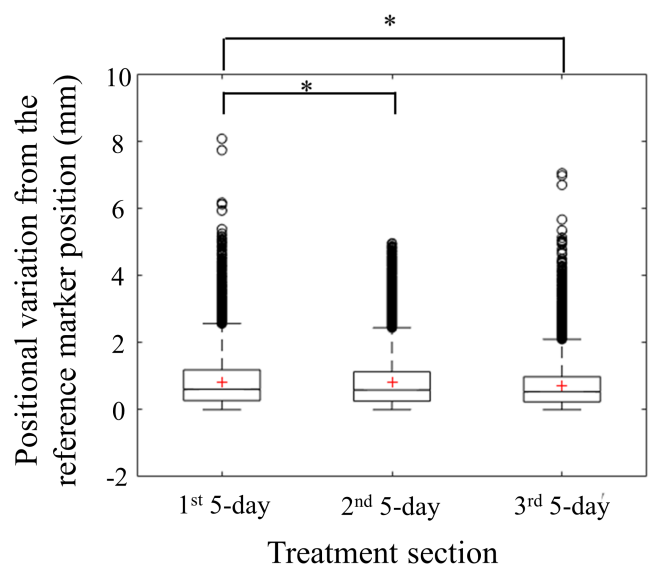

(b) Y-axis

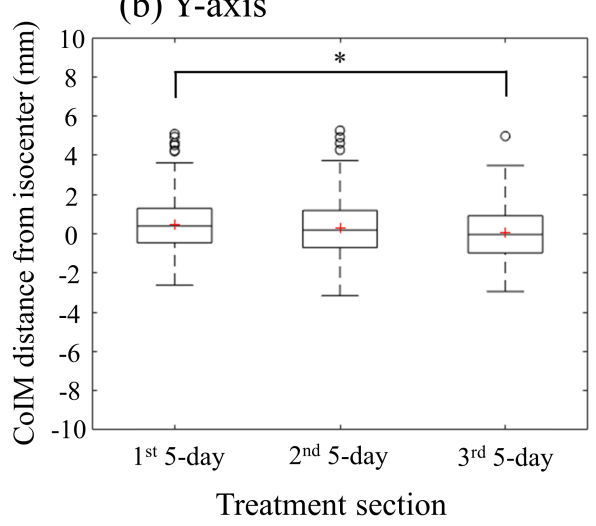

Treatment section

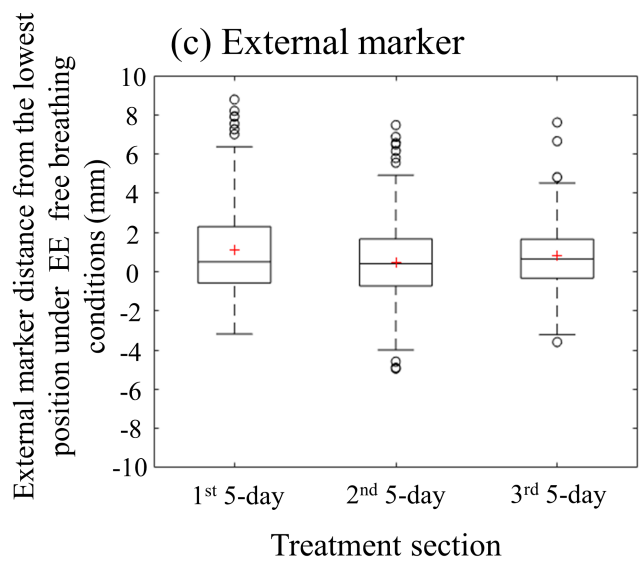

Treatment section (e) External marker

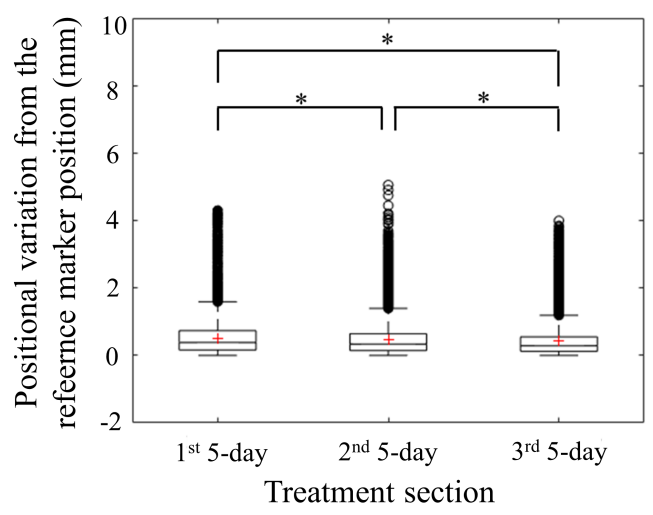

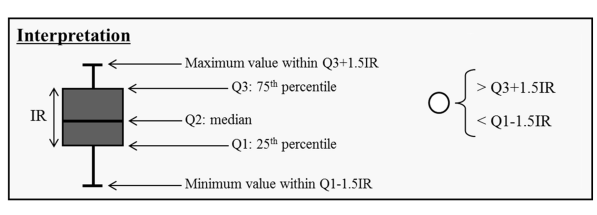

Red cross; mean value $* ; p<0.05$

\section{Figure 4}

Box-and-whisker plots of the inter-fractional subanalyses of positional repeatability among multiple $\mathrm{BH}$ sessions and positional variation within $\mathrm{BH}$ sessions, for all patients. The positional repeatability among multiple BH sessions for the CoIM on the X-axis (a) and on the $\mathrm{Y}$-axis (b), and for the external marker (c). The positional variation within BH sessions for the CoIM on the $\mathrm{Y}$-axis (d), and for the external marker (e). The SI direction for patients on fluoroscopic images corresponded to the $Y$-axis; the $X$-axis was perpendicular to the $\mathrm{Y}$-axis. 

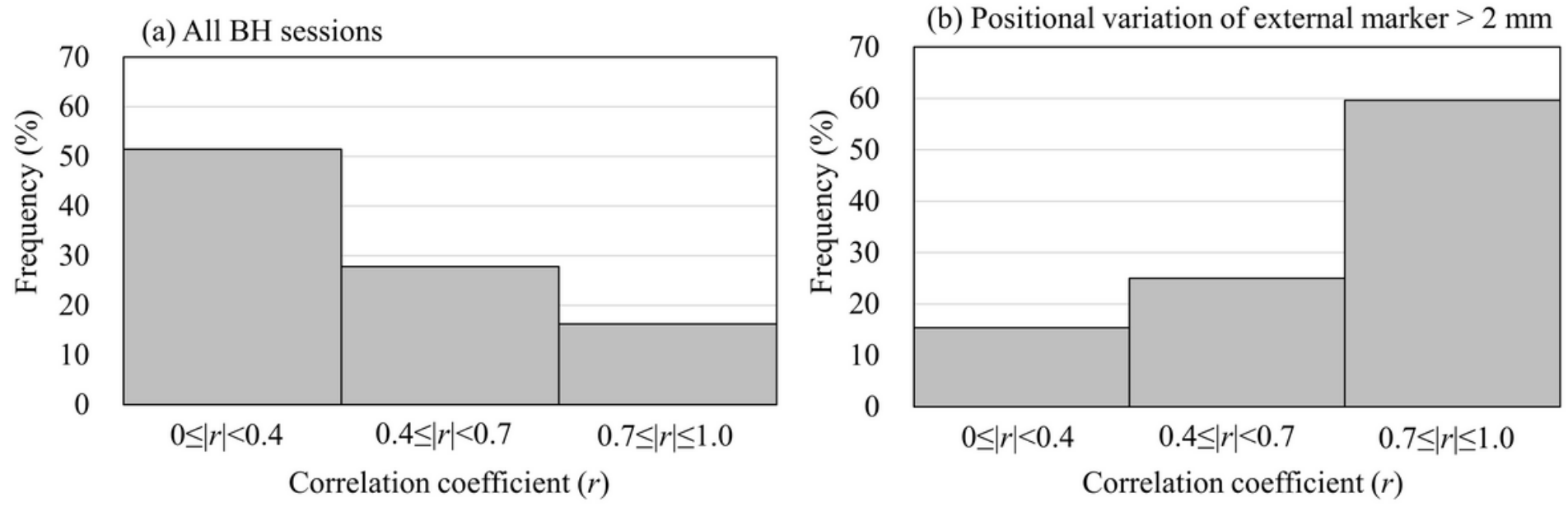

(c) Relationship between the regression coefficient and the correlation coefficient

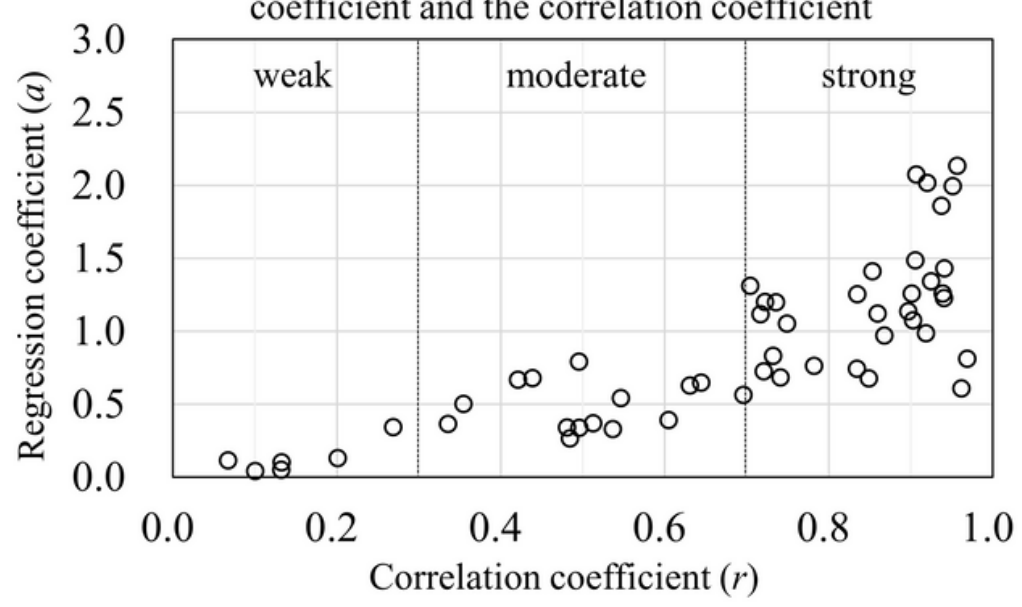

Figure 5

Correlation between the internal and external markers. The histogram of the correlation coefficients for all $\mathrm{BH}$ sessions (a), and that of the sessions where the positional variation of the external marker relative to the reference position after $\mathrm{BH}$ was greater than $2 \mathrm{~mm}$ (b). The relationship between the regression coefficient (a) and correlation coefficients $(r)$ in cases where the positional variation of the external marker exceeded $2 \mathrm{~mm}$ (c). 

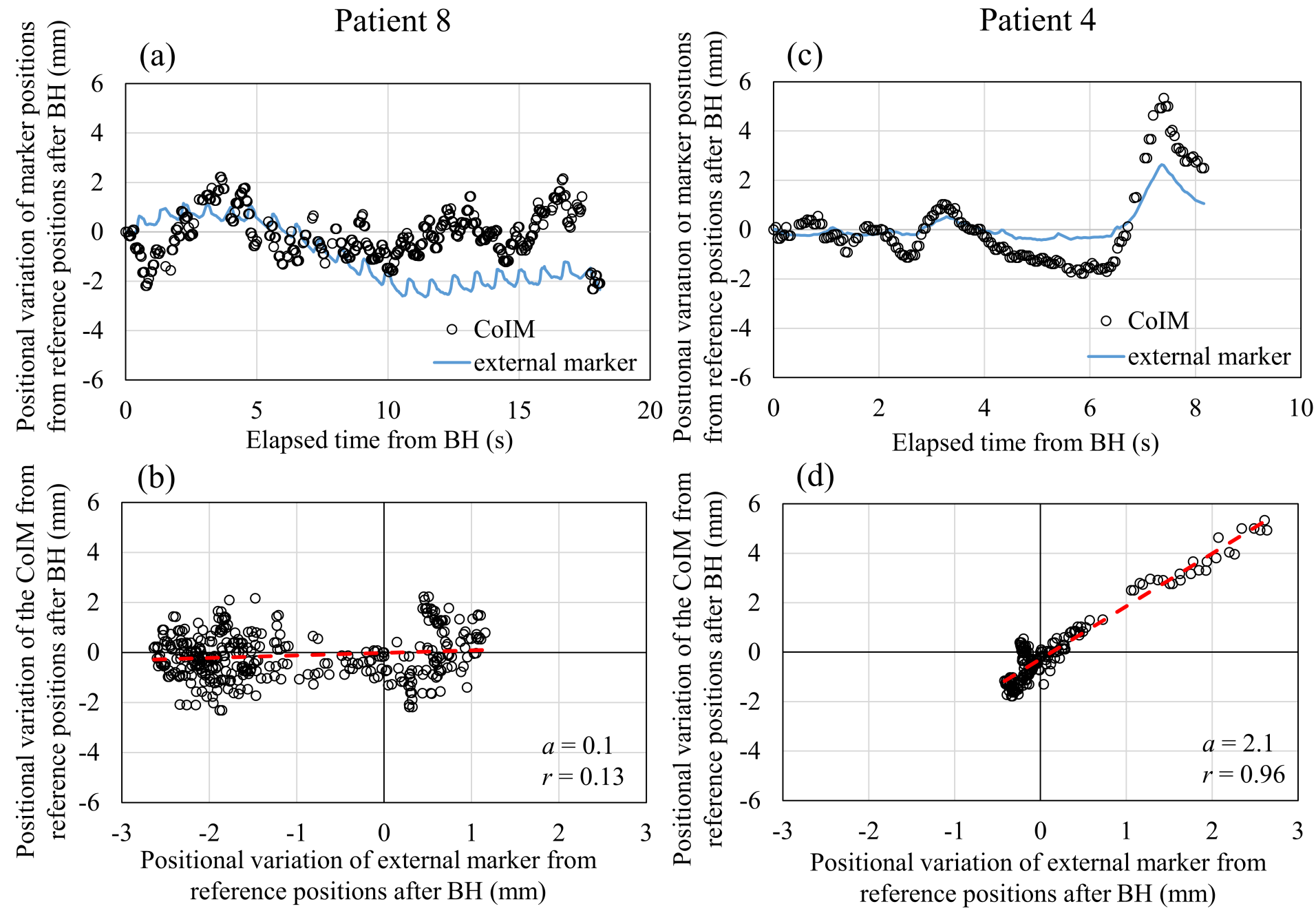

Figure 6

A case (patient 8) showing a weak correlation between the two marker motions ( $a, b)$, and a case (patient 4) showing a strong correlation between the two marker motions (c, d). 